\title{
Foot Self-care Practices Among Filipino American Women with Type 2 Diabetes Mellitus
}

Deovina Nasis Jordan · James Lowell Jordan

Received: November 3, 2010 / Published online: January 31, 2011

(c) The Author(s) 2011. This article is published with open access at Springerlink.com

\section{ABSTRACT}

Introduction: To determine the foot self-care practices performed by Filipino American (FA) women with type 2 diabetes mellitus (DM). Method: The Summary of Diabetes Self Care Activities - Revised and Expanded measure was administered to 118 FA adult female immigrants with type 2 DM. Results: Younger FA women ( $<65$ years), participants with higher education, those who immigrated to the United States (US) at younger ages, and participants diagnosed with type $2 \mathrm{DM}$ at younger ages reported they washed their feet every day during the past week. Moreover, FA women who immigrated to the US at younger ages and participants who were diagnosed with the disease at younger ages reported that they dried their feet (in between toes) daily during the previous week. Further, FA women who were diagnosed with type $2 \mathrm{DM}$ at younger ages were more likely to report that they checked their feet every day during the

Deovina Nasis Jordan $(\varangle) \cdot$ James Lowell Jordan Department of Nursing, 5 West, Ronald Reagan UCLA Medical Center 5354, 757 Westwood Plaza, Los Angeles, CA 90095-7404, USA. Email: dnjordan@ucla.edu, and djordan@mednet.ucla.edu past week, when compared with participants who were diagnosed with the disease at older ages. Finally, most FA women did not inspect the inside of their shoes. Conclusion: Foot selfcare practices were less frequently performed by older FA women with type $2 \mathrm{DM}$ ( $\geq 65$ years), making them more prone to the development of foot problems such as ulcers, infections, and disfigurations. Optimum foot self-care practices must be encouraged in older FA women to prevent such foot problems, and subsequent amputations.

Keywords: diabetic foot; diabetic neuropathy; Filipino immigrants; foot infections; foot ulcers; type 2 diabetes

\section{INTRODUCTION}

One in six individuals with type 2 diabetes mellitus (DM) will develop a foot ulcer in his/her lifetime; $7 \%$ have foot ulcers at any given time, and $15 \%$ of foot ulcers end in amputation. ${ }^{1-3}$ Foot ulcers are the most expensive complication of type $2 \mathrm{DM}$, accounting for about $20 \%$ of the costs and $30 \%$ of hospital admissions associated with treating persons with type 2 DM. ${ }^{1,4,5}$ Compliance with proper foot care reduces the incidence of 
foot ulcers, particularly among persons with reduced severity of neuropathy. ${ }^{6}$ A study by Apelqvist and Agardh ${ }^{7}$ found that $25 \%$ of clients who had foot ulcers underwent amputation, and $13 \%$ died. ${ }^{7}$ Moreover, as many as $85 \%$ of lower extremity amputations can be avoided by prompt and appropriate treatment of diabetic foot wounds and ulcers by healthcare providers, as well as optimum foot self-care practices by individuals affected by the condition. ${ }^{8-11}$ Unfortunately, many persons with type $2 \mathrm{DM}$ do not receive adequate foot care instruction from their healthcare providers and, as such, do not perform routine foot examinations. ${ }^{12}$

Optimum foot self-care practices include daily inspection of feet and inside of shoes; daily washing of feet and careful drying afterwards (especially the areas inbetween the toes); not walking barefoot; wearing proper footwear (use of therapeutic shoes with pressure-relieving insoles and not sandals or poorly fitting shoes); cutting toenails straight across; not removing ingrown toenails, calluses, corns, or warts oneself; avoiding the use of antiseptic lotions and adhesive tape on the feet; obtaining prompt professional healthcare treatment if new lesions, cuts, bruises, and wounds are noted; and screening of feet regularly, or at least once annually, by a healthcare provider to identify feet at risk for diabetic foot complications. ${ }^{13-17}$ Many persons with type $2 \mathrm{DM}$, however, neglect their foot care $^{18}$ and regular foot examinations because of painless neuropathic feet, which cause them to ignore even the more serious of foot injuries. ${ }^{1}$ Such persons may also cause additional foot damage by subjecting their feet to further trauma; while persons without peripheral neuropathy tend not to place weight upon an injured foot, those with peripheral neuropathy continue to do so. ${ }^{19}$

One population greatly affected by diabetic foot care is the Filipino American (FA) community in the United States, which is a group at high risk for developing type $2 \mathrm{DM}$ and its complications. ${ }^{20-23}$ However, in spite of the large number of Filipino immigrants in the United States, many of whom are older females, little scholarly research has been conducted on them and their health. Currently, no articles dealing with the foot self-care practices of FAs, specifically older FA women with type 2 DM, appear to exist, even though this ethnic group's mortality rate from DM is three times higher than that of the white population. ${ }^{24}$

This research study was conducted to examine the foot self-care practices performed by FA women with type $2 \mathrm{DM}$ in order to contribute knowledge that will help to optimize their health, and improve their overall quality of life.

\section{MATERIALS AND METHODS}

This research study involved 118 FA women residing in Los Angeles and Orange Counties, California, USA. The sociodemographic data were gathered using the Baseline Instrument for Filipino Americans with Type 2 Diabetes Mellitus (BIFAD) instrument. ${ }^{25}$ The FA women included in the study were: (1) medically diagnosed with type $2 \mathrm{DM}$, (2) older than 30 years, (3) capable of reading and/or speaking in English, (4) southern California residents, (5) lacking disabilities (eg, physical, mental) that would prevent them from participating and completing the study procedures, and (6) were first-generation immigrants to the United States from the Philippines.

All participation was voluntary and all responses (and participation) were confidential, as per the study approval by the University of California Los Angeles Institutional Review Board. If participants met the inclusion criteria, no additional selection criteria were used to determine either eligibility or inclusion of their 
responses in the database. Recruitment and data collection proceeded over a continuous period of 3.5 months.

The foot self-care data were collected using the Summary of Diabetes Self Care ActivitiesRevised and Expanded (SDSCA-R\&E) measure developed by Toobert et al. ${ }^{26}$ Diagnoses of type $2 \mathrm{DM}$ and foot self-care practices were obtained from self-reports. The SDSCA-R\&E measure has four questions addressing recommended foot selfcare among persons with type $2 \mathrm{DM}$. They are: (1) On how many of the last 7 days did you wash your feet? (2) On how many of the last 7 days did you dry between your toes after washing? (3) On how many of the last 7 days did you check your feet? and (4) On how many of the last 7 days did you inspect the insides of your shoes? The data were collected by the principal investigator, who was able to answer any questions the participants had about the study.

Statistical analyses were performed using SPSS software, V.15 (SPSS, Chicago, IL, USA). ${ }^{27}$ Descriptive statistics (mean, standard deviation), using Student's $t$ tests, were calculated on the FA women's age groupings, age, education, age at immigration to the United States, and age when diagnosed with type $2 \mathrm{DM}$ ( $<65$ years of age and $\geq 65$ years of age). Likewise, descriptive statistics (frequency, percentage) using one-way analysis of variance were computed for performance of foot self-care practices.

The days per week were clustered in duration as "0 days" (foot self-care was never performed during the past week), " 1 to 3 days" (representing foot self-care performed on fewer than $50 \%$ of days in the past week), " 4 to 6 days" (indicating foot self-care performed more than $50 \%$ of days in the past week), and "7 days" (foot self-care done every day during the past week). Further, the foot self-care data were then analyzed using Spearman's rank correlation to test the direction and strength of the relationships between the dependent variables of foot self-care practices and the independent variables of age, education, age at immigration to the United States, and age when diagnosed with type $2 \mathrm{DM}$.

\section{RESULTS}

Descriptive statistics on younger ( $<65$ years of age) and older ( $\geq 65$ years of age) FA women with type $2 \mathrm{DM}$ are presented in Table 1 . The mean age of the participants was 68 years. As for education, their mean number of years of schooling was 12 years. All participants were immigrants from the Philippines. They immigrated to the United States at a mean age of 49 years and were diagnosed with type $2 \mathrm{DM}$ at a mean age of 57 years. The frequencies and percentages that the women performed foot selfcare practices are provided in Table 2 .

Most FA women reported that they washed their feet, dried their feet (inbetween the toes), and checked their feet every day during the previous week. However, less than one-third of the women inspected the insides of their shoes every day during the past week; most of them had not inspected the insides of their shoes during the previous week. The foot self-care practices and predictors of age, education, age at immigration, and age when diagnosed with type $2 \mathrm{DM}$ are presented in Table 3.

\section{Washing of Feet}

Younger FA women with type 2 DM were more likely to report that they washed their feet every day during the past week, while older participants reported that they washed their feet less often. Likewise, FA women with higher educational attainment reported that they washed their feet more frequently than those participants with lower educational attainment. Moreover, FA women who immigrated at a younger age were 
Table 1. Descriptive statistics of younger ( $<65$ years of age) and older ( $\geq 65$ years of age) Filipino American women with type 2 diabetes mellitus (DM) $(n=118)$; significance determined using Student's $t$ test.

\begin{tabular}{lccc}
\hline Variable & $\begin{array}{c}\text { Younger participants } \\
(n=39)\end{array}$ & $\begin{array}{c}\text { Older participants } \\
(n=79)\end{array}$ & $\begin{array}{c}\text { Overall } \\
(n=118)\end{array}$ \\
\hline Age, years & $56 \pm 8.2$ & $74 \pm 4.7$ & $68.3 \pm 10.5$ \\
Education, ${ }^{*}$ years & $13 \pm 2.4$ & $11 \pm 3.3$ & $11.8 \pm 3.1$ \\
Age at immigration to United States, $\dagger$ years & $35 \pm 11.3$ & $55 \pm 9.6$ & $48.7 \pm 13.9$ \\
Age when diagnosed with type 2 DM, $\dagger$ years & $46 \pm 8.2$ & $62 \pm 11.0$ & $56.7 \pm 12.6$ \\
\hline
\end{tabular}

All data mean \pm standard deviation (SD).

${ }^{*} P<0.01$.

$\dagger P<0.001$.

more likely to report that they washed their feet 7 days during the past week, while those who immigrated when they were older did not perform the foot self-care practice regularly. Furthermore, FA women who were diagnosed with type 2 $\mathrm{DM}$ at a younger age were more likely to report that they washed their feet every day during the prior week and, therefore, did so more often than those participants who were diagnosed with type $2 \mathrm{DM}$ at an older age (Table 3).

\section{Drying of Feet (Inbetween Toes) After Washing}

FA women who immigrated to the United States at a younger age were more likely to report that they dried their feet inbetween the toes every day after washing during the past week than participants who immigrated to the United States at an older age. Likewise, FA women who were diagnosed with type $2 \mathrm{DM}$ at a younger age were more likely to report that they dried their feet 7 days during the last week, while those participants who were diagnosed with type $2 \mathrm{DM}$ when they were older dried their feet less often (Table 3).

\section{Checking of Feet}

FA women who were younger when they were diagnosed with type 2 DM were more likely to report that they checked their feet every day during the previous week, while those participants who were older when diagnosed with the condition did not check their feet, or checked their feet less regularly (Table 3 ).

\section{Inspecting the Inside of Shoes}

In all, $32 \%$ of the participants reported that they did not inspect the insides of their shoes

Table 2. Frequencies and percentages of performance of foot self-care practices among Filipino American women with type 2 diabetes mellitus (DM) $(n=118)$.

\begin{tabular}{lcccc}
\hline & \multicolumn{4}{c}{ Frequency (days per week) } \\
\cline { 2 - 5 } Foot self-care practice & $\mathbf{0}$ & $\mathbf{1 - 3}$ & $\mathbf{4 - 6}$ & $\mathbf{7}$ \\
\hline Washing of feet, $n(\%)$ & $1(0.9)$ & $31(26.3)$ & $24(20.3)$ & $62(52.5)$ \\
Drying inbetween toes, $n(\%)$ & $12(10.2)$ & $26(22.0)$ & $23(19.5)$ & $57(48.3)$ \\
Checking of feet, $n$ (\%) & $15(12.7)$ & $23(19.5)$ & $16(13.6)$ & $64(54.2)$ \\
Inspecting inside of shoes, $n(\%)$ & $38(32.2)$ & $27(22.9)$ & $19(16.1)$ & $34(28.8)$ \\
\hline
\end{tabular}


Table 3. Relationships of foot self-care practices and predictors among Filipino American women with type 2 diabetes mellitus (DM) ( $n=118)$ (examined using Spearman's rank correlation coefficient).

\begin{tabular}{lcccc}
\hline & \multicolumn{4}{c}{ Predictor } \\
\cline { 2 - 5 } Foot self-care practice & Age & $\begin{array}{c}\text { Education, } \\
\text { years }\end{array}$ & $\begin{array}{c}\text { Age at } \\
\text { immigration to } \\
\text { United States, years }\end{array}$ & $\begin{array}{c}\text { Age when } \\
\text { diagnosed with } \\
\text { type 2 DM, years }\end{array}$ \\
\hline Washing of feet & $-0.247, P=0.007$ & $-0.224, P=0.015$ & $-0.289, P=0.002$ & $-0.405, P=0.000$ \\
Drying inbetween toes & $0.051, P=0.582$ & $0.118, P=0.203$ & $-0.047, P=0.611$ & $0.038, P=0.681$ \\
Checking of feet & $-0.168, P=0.069$ & $-0.035, P=0.709$ & $-0.165, P=0.074$ & $-0.372, P=0.000$ \\
Inspecting inside of shoes & $0.052, P=0.579$ & $-0.051, P=0.582$ & $-0.003, P=0.975$ & $0.006, P=0.947$ \\
\hline
\end{tabular}

Correlation coefficients (two-tailed) and probabilities are shown.

during the past week. About 23\% reported that they performed the foot self-care practice approximately 1 to 3 days during the last week, while slightly over $16 \%$ reported that they performed the foot self-care practice on about 4 to 6 days during the past week. Almost 29\% of the participants reported that they inspected the insides of their shoes every day during the previous week (Table 2). There was no relationship between the foot self-care practice of inspecting the inside of shoes and the predictors of age, education, age at immigration to United States, and age when diagnosed with type $2 \mathrm{DM}$ (Table 3).

\section{DISCUSSION}

This research study reports on four major findings. Firstly, younger FA women, participants with higher educational attainment, those who were younger when they immigrated to the United States, and participants who were diagnosed with type $2 \mathrm{DM}$ at a younger age were more likely to report that they washed their feet every day during the past week. Secondly, FA women who immigrated to the United States at a younger age and those who were diagnosed with the disease at a younger age were more likely to report that they dried their feet (inbetween the toes) daily during the last week. Thirdly, participants who were younger when they were diagnosed with type $2 \mathrm{DM}$ were more likely to report that they checked their feet 7 days during the past week. Finally, while almost one-third (29\%) of the participants reported that they inspected the insides of their shoes every day during the previous week, there was no relationship between inspecting the inside of shoes and the predictors of age, education, age at immigration to United States, and age when diagnosed with type $2 \mathrm{DM}$.

Poor diabetes foot care knowledge and, consequently, lack of foot self-care (eg, infrequent washing of feet, drying of feet inbetween toes, checking of feet, and inspecting the inside of shoes) have been associated with an inability to sense minor injuries to the foot and a tendency toward ulceration. When individuals with type $2 \mathrm{DM}$ fail to practice proper foot self-care, the likelihood of ulcers increases along with a higher risk for subsequent amputation and/or mortality. ${ }^{6,14,28-30}$ Since individuals with type 2 DM may not be able to sense foot injuries (eg, from foreign objects lodged within their shoes) during or after its occurrence, frequent checking of the feet becomes crucial. For those persons who are at higher risk for foot injury, such as the older FA female participants in this study, 
education and treatment interventions by healthcare providers become even more critical; failure to do so may lead to situations where the foot problems are not only costly to treat, but may also be impossible to heal (leading to amputations).

Minor trauma can also develop from repetitive, moderate pressure, ${ }^{15}$ such as from wearing improperly fitting shoes or sandals. Because individuals with type $2 \mathrm{DM}$ also suffer from peripheral neuropathy, frequent checking of the inside of shoes and use of diabetic footwear is highly recommended. Adequate, acceptable, and reasonably priced footwear should be combined with good foot self-care practices. ${ }^{31}$ This research study found that participants with lower educational attainment inspected the insides of their shoes more often (7 days during the past week) than participants with higher educational attainment. The reason(s) for this finding, however, is not known. Some explanations for nonperformance or infrequent performance of proper foot self-care practices are major depression and obesity; 32 eye problems, decreased mobility, and loss of flexibility; ${ }^{9}$ lack of insurance and other financial barriers to healthcare services; ${ }^{33}$ and a lack of knowledge on foot care. ${ }^{34}$ However, this research study did not investigate the reasons for nonperformance or infrequent performance of proper foot self-care practices in FA women; hence, further inquiry is recommended.

\section{Limitations of the Study and Directions for Future Research}

Since this research study only dealt with foot self-care practices, information on access to healthcare services and personal experiences pertaining to healthcare were not obtained. It would be interesting to know whether older FA women had the same access to healthcare services as younger FA women. Chronic social stressors, such as discrimination, can lead to occurrence of chronic health problems. Treating chronic health problems, therefore, should entail not only treating the health condition, but also dealing with the perception of discrimination and/or stressors in the client's environment. ${ }^{35}$ These issues are very important, and will need to be investigated further.

\section{CONCLUSION}

Proper foot self-care practices were less likely to be performed by older FA women with type $2 \mathrm{DM}$, making them more prone to the development of foot problems, such as ulcers, infections, and disfigurations. It is not known why this phenomenon was observed in this segment of the population. Hence, more research is needed to determine the reasons for this observation and to develop the best ways to increase optimum foot self-care behaviors in this vulnerable population. Preventive practices must be emphasized in older FA women with type $2 \mathrm{DM}$ to prevent foot ulcers, foot infections, and subsequent amputations.

\section{ACKNOWLEDGMENTS}

The authors of this manuscript have no conflicts of interest, financial or otherwise, to disclose. The research was funded via personal means. Deovina Nasis Jordan is the guarantor for this article, and takes responsibility for the integrity of the work as a whole.

Open Access. This article is distributed under the terms of the Creative Commons Attribution Noncommercial License which permits any noncommercial use, distribution, and reproduction in any medium, provided the original author(s) and source are credited. 


\section{REFERENCES}

1. Young MJ. Foot problems in diabetics. In: Pickup JC, Williams G, eds. Textbook of Diabetes 1 . Malden, MA: Blackwell Science; 2003:57.1-57.19.

2. Boulton AJM, Kirsner RS, Vileikyte L. Neuropathic diabetic foot ulcers. N Engl J Med. 2004;351:48-55.

3. Ramsey SD, Newton K, Blough D, et al. Incidence, outcomes, and cost of foot ulcers in patients with diabetes. Diabetes Care. 1999;22:382-387.

4. Gibbons G, Eliopolos G. Infection of the diabetic foot. In: Kozak G, Hoar CJ, Rowbotham J, Wheelock F, Gibbons G, Campbell D, eds. Management of Diabetic Foot Problems. Philadelphia, PA: Saunders; 1984:97-102.

5. Williams R, Airey M. The size of the problem: economic aspects of foot problems in diabetes. In: Boulton AJM, Connor H, Cavanagh PR, eds. The Foot in Diabetes. 3rd edition. Hoboken, NJ: Wiley; 2000:3-17.

6. Calle-Pascual AL, Durán A, Benedí A, et al. A preventive foot care programme for people with diabetes with different stages of neuropathy. Diabetes Res Clin Prac. 2002;57:111-117.

7. Apelqvist J, Agardh CD. The association between clinical risk factors and outcome of diabetic foot ulcers. Diabetes Res Clin Prac. 1992;18:43-35.

8. American Diabetes Association. 2001 Vital Statistics. Alexandria, VA: American Diabetes Association; 2001.

9. Green ME, Aliabadi Z, Green BT. Diabetic foot: evaluation and management. Southern Med J. 2002;95:95-101.

10. Price P. The diabetic foot: quality of life. Clin Infect Dis. 2004;39(suppl. 2):S129-S131.

11. Strauss MB. Surgical treatment of problem foot wounds in patients with diabetes. Clin Orthop Rel Res. 2005;439:91-96.

12. De Berardis G, Pellegrini F, Franciosi M, Belfiglio M, Di Nardo B, Greenfield S. Are type 2 diabetic patients offered adequate foot care? The role of physician and patient characteristics. J Diabetes Complications. 2005;19:319-327.

13. American Diabetes Association. Foot care guidelines. Diabetes Educ. 2004;30:384-385.
14. Frykberg RG. Diabetic foot ulcers: pathogenesis and management. Am Fam Physician. 2002;66:16551622.

15. Frykberg RG, Hayden CT. A summary of guidelines for managing the diabetic foot. Adv Skin Wound Care. 2005;18:209-214.

16. Johnson M, Newton P, Goyder E. Patient and professional perspectives on prescribed therapeutic footwear for people with diabetes: a vignette study. Patient Educ Couns. 2006;64:167-172.

17. Martinez NC, Tripp-Reimer T. Diabetes nurse educators' prioritized elder foot care behaviors. Diabetes Educ. 2005;31:858-868.

18. Safford MM, Russell L, Suh DC, Roman S, Pogach L. How much time do patients with diabetes spend on self-care? J Am Board Fam Pract. 2005;18:262270.

19. Boulton AJM, Armstrong DG. The diabetic foot. In: DeFronzo RA, Ferrannini E, Keen H, Zimmet P, eds. International Textbook of Diabetes Mellitus. 3rd edition. Hoboken, NJ: Wiley. 2006:179-195.

20. Araneta MRG, Barrett-Connor E. Ethnic differences in visceral adipose tissue and type 2 diabetes: Filipino, African-American, and white women. Obes Res. 2005;13:1458-1465.

21. Araneta MRG, Barrett-Connor E. Subclinical coronary atherosclerosis in asymptomatic Filipino and White women. Circulation. 2004;110:28172823.

22. Araneta MR, Morton DJ, Lantion-Ang L, et al. Hyperglycemia and type 2 diabetes among Filipino women in the Philippines, Hawaii, and San Diego. Diabetes Res Clin Pract. 2006;71:306-312.

23. Langenberg C, Marmot M, Araneta MRG, BarrettConnor E, Bergsrom J. Diabetes and coronary heart disease in Filipino American women. Diabetes Care. 2007;30:535-541.

24. Hawaii State Department of Health. Hawaii Diabetes Report, 2004. Figure 9. Available at: http:// www.hawaii.gov/health/family-child-health/ chronic-disease/diabetes/pdf/diabetesreport.pdf 2004. Accessed May 18, 2006.

25. Jordan DN. Comorbidities, Perceptions, Self-Care Behaviors, and Foot Self-Care Practices of Filipino American Adults with Type 2 Diabetes Mellitus. Doctoral Dissertation: University of California, Los Angeles, 2008. 
26. Toobert DJ, Hampson SE, Glasgow RE. The summary of diabetes self-care activities measure. Diabetes Care. 2000;23:943-950.

27. SPSS, Inc. SPSS PC-software, version 15. Chicago, IL: SPSS, Inc.; 2006.

28. Pollock RD, Unwin NC, Connolly V. Knowledge and practice of foot care in people with diabetes. Diabetes Res Clin Pract. 2004;64:117-122.

29. Rathur HM, Boulton AJM. The neuropathic diabetic foot. Nat Clin Pract Endocrinol Metab. 2007;3: 14-25.

30. $\mathrm{Wu} \mathrm{S}$, Armstrong DG. Risk assessment of the diabetic foot and wound. Int Wound J. 2005;2: 17-24.

31. Reiber GE, Smith DG, Wallace CM, et al. Footwear used by individuals with diabetes and a history of foot ulcer. J Rehabil Res Dev. 2002;39:615-622.
32. Maraldi C, Volpato S, Penninx BW, Yaffe K, Simonsick EM, Strotmeyer ES. Diabetes mellitus, glycemic control, and incident depressive symptoms among 70- to 79-year-old persons: the health, aging, and body composition study. Arch Intern Med. 2007;167:1137-1144.

33. Ro M. Moving forward: addressing the health of Asian American and Pacific Islander women. Am J Public Health. 2002;92:516-519.

34. Han PY, Ezquerro R, Pan K, Hwang S, Chung $\mathrm{Y}$, Lu J. Comorbidities associated with diabetic complications among Asian Americans in Southern California. J Am Podiatr Med Assoc. 2003;93:37-41.

35. Gee GC, Chen J, Spencer MS, See S, Kuester OA, Takeuchi TD. Social support as a buffer for perceived unfair treatment among Filipino Americans: differences between San Francisco and Honolulu. Am J Public Health. 2006;96:677-684. 